\title{
THE NOVEMBER MEETING IN LAFAYETTE
}

The four hundred eighty-sixth meeting of the American Mathematical Society was held at Purdue University, West Lafayette, Indiana, on November 28-29, 1952. There were approximately 130 registrations, including the following 86 members of the Society:

D. N. Arden, Nachman Aronszajn, W. L. Ayres, P. T. Bateman, P. O. Bell, R. L. Blair, Leonard Bristow, E. F. Buck, R. C. Buck, I. W. Burr, J. W. Carr, Lamberto Cesari, Y. W. Chen, J.A. Dieudonné, C. L. Dolph, John Dyer-Bennet, W. E. Edington, B. J. Eisenstadt, J. G. Elliott, Paul Erdös, Chester Feldman, D. A. Flanders, J. S. Frame, C. G. Fry, R. E. Fullerton, Murray Gerstenhaber, Casper Goffman, Michael Golomb, A. W. Goodman, S. H. Gould, G. H. Graves, L. M. Graves, R. L. Graves, H. E. H. Greenleaf, J. K. Hale, P. C. Hammer, Sister M. Agnes Hatke, C. T. Hazard, L. L. Helms, Melvin Henriksen, J. G. Hocking, H. K. Hughes, Ralph Hull, P. E. Irick, W. E. Jenner, Meyer Jerison, Samuel Kaplan, M. W. Keller, J. H. B. Kemperman, C. F. Kossack, J. R. Lee, Y. L. Luke, J. D. McKnight, Jr., J. M. Mitchell, M. A. Moore, R. H. Niemann, Rufus Oldenburger, D. B. Owen, Sam Perlis, J. C. Polley, L. E. Pursell, C. R. Putnam, Gustave Rabson, Tibor Rad6, W. T. Reid, B. V. Ritchie, P. C. Rosenbloom, Arthur Rosenthal, E. H. Rothe, W. C. Sangren, Lowell Schoenfeld, W. T. Scott, M. E. Shanks, M. F. Smiley, A. H. Smith, R. B. Stone, E. W. Titt, E. A. Trabant, N. H. Vaughan, G. L. Walker, M. S. Webster, C. P. Wells, George Whaples, Oswald Wyler, L. C. Young, J. W. T. Youngs.

By invitation of the Committee to select Hour Speakers for Western Sectional Meetings, Professor Nachman Aronszajn of the University of Kansas addressed the Society on Approximation methods in eigenvalue problems at 2:00 P.M. on Friday. The speaker was introduced by Professor Tibor Rado, and the interest in the topic is attested to by the large number of contributed papers on the program which followed Professor Aronszajn's address and dealt with the same field.

There were sessions for contributed papers at 3:30 P.M. on Friday and at 9:30 A.M. and 11:00 A.M. on Saturday. Presiding officers for these sessions were Professors Arthur Rosenthal, W. T. Reid, Ralph Hull, and L. C. Young.

There was a dinner for members of the Society and their guests at the Purdue Memorial Union on Friday night, followed by open house in the homes of the various members of the local department. The Society takes this opportunity to record its pleasure and appreciation.

Abstracts of papers presented at the meeting are recorded below. Papers with abstract numbers followed by " $t$ " were presented by 
title. Paper number 88 was read by Dr. Sangren, number 95 by Professor Young.

\section{Algebra AND Theory of Numbers \\ 82. Melvin Henriksen: On the prime ideals of the ring of entire func- tions.}

The existence of nonmaximal ideals of the ring $R$ of entire functions was pointed out to the author by Professor I. Kaplansky. If we let $0_{n}(f)$ be the multiplicity of the $n$th zero of $f$, these ideals are characterized as the prime ideals $P$ such that $0_{n}(f)$ is unbounded, for all $f \in P$. Any nonmaximal prime ideal of $R$ is free (for definitions, see Pacific Journal of Mathematics vol. 2, pp. 179-184). Each prime ideal is contained in a unique maximal (free) ideal $M$. The class $P$ of all prime ideals contained in $M$ is linearly ordered under set inclusion, and $P^{*}=\bigcap_{k=1}^{\infty} M^{k}$ is the largest nonmaximal ideal of $P$. The residue class ring $R / P$ is a valuation ring and is Noetherian if and only if $P=P^{*}$. The residue class ring $R / P^{*}$ is isomorphic to the ring of all formal power series over the complex field. The structure theory of Cohen (Trans. Amer. Math. Soc. vol. 59, pp. 54-106) of complete local rings is used. (Received December 4, 1952.)

\section{George Whaples: Additive polynomials.}

Additive polynomials are those of form $f(x)=\sum \alpha_{\nu} x^{p^{\nu}}$ over a field $k$ of characteristic $p$. They are characterized by the identity $f(x+y) \equiv f(x)+f(y)$, form a ring under addition and composition, and have been studied by O. Ore (Trans. Amer. Math. Soc. vol. 35 (1933) pp. 559-584 and vol. 36 (1934) pp. 243-274) for arbitrary $k$ and for $k$ a Galois field. This paper assumes $k$ has characteristic $p$, has no inseparable extensions, and has for each $n$ at most one extension of degree $n$ (exactly one for $n=p$ ) in its algebraic closure. There are many such fields not absolutely algebraic. Main results $\left(k^{+}=\right.$additive group of $k, f(x)=$ an a.p.): 1. (Index of $f\left(k^{+}\right)$in $\left.k^{+}\right)=$number of zeros of $f(x)$ in $k \leqq$ degree $f(x)$. 2. Every group between $f\left(k^{+}\right)$and $k^{+}$is set of values of some a.p. 3. $k^{+}$can be topologized by taking the sets of values of the a.p. as neighborhoods of 0 . Under the induced uniform topology on the ring $C$ of continuous endomorphisms of $k^{+}$, the a.p. are everywhere dense in $C$. (Received October 15, 1952.)

\section{Analysis}

\section{R. C. Buck: Generalized asymptotic density.}

Let $\mu_{n}$ be a sequence of measures on $X$, and $K_{1} \subset K_{2} \subset \cdots$ be a sequence of sets of finite measure exhausting $X$ such that (i) $\lim _{n} \mu_{n}\left(K_{j}\right)=0$ for each $j$, (ii) $\mu_{n}(X)=1$ for all $n$, (iii) the support of each $\mu_{n}$ lies in some $K_{j}$. Write $A \dot{C} B$ when there is a $j$ such that $A-K_{j} \subset B-K_{j}$. A measurable set $S$ has density $D(S)=d$ if $d=\lim \mu_{n}(S)$. Theorem: if $\left\{A_{n}\right\}$ is a sequence of mutually disjoint sets having density, there is a set $S$, unique up to sets of zero density, such that $D(S)=\sum D\left(A_{n}\right)$, and such that for each $n, S \supset \bigcup_{1}^{n} A_{k}$. Application: let $f(x)$ be real-valued, measurable, and bounded below on $X$. For any $\epsilon>0$, let $f(x)>-\epsilon$ on a set of unit density, and suppose that $\lim _{n \rightarrow \infty} \int f d \mu_{n}=0$. Then, there is a set $E$ of zero density such that as $x \rightarrow \infty$ in $X-E$, $f(x) \rightarrow 0$. The topology at $\infty$ in $X$ is that defined by $X-K_{n}$. (Received October 16, 1952.)

85t. R. C. Buck: On limit points of complex sequences. 
Let $\left\{s_{n}\right\}$ be a bounded complex sequence, and let $C$ be its core, the union of the closed convex hulls of the sets $\left\{s_{n}, s_{n+1}, \cdots\right\}$. Any extreme point of $C$ will be called an outer limit point of $\left\{s_{n}\right\}$, generalizing the upper and lower limits of a real sequence. Theorem: if $\left\{s_{n}\right\}$ is Cesaro summable to one of its outer limit points $p$, then except for a subsequence of zero density, $\left\{s_{n}\right\}$ converges to $p$. The theorem remains true if $\left\{s_{n}\right\}$ is allowed to be unbounded, provided that $p$ is a regular extreme point of $C$ for which there is a supporting line touching $C$ only at $p$. (Received October $16,1952$. )

\section{Lamberto Cesari: On a geometrical proof of the equality $V(S)$} $=L(S)$ for continuous parametric surfaces.

Let $S: p=p(w), w=(u, v) \in Q, p=(x, y, z) \in E_{3}$ denote any continuous parametric surface in $E_{3}$, i.e., any continuous mapping from a set $Q$ of the $w$-plane into $E_{3}$. Then the projections of $S$ on the coordinate planes are plane mappings $T_{i}, i=1,2,3$. Let $L(S)$ and $V(S)$ be the Lebesgue area and the so-called Geocze area of $S$. The equality $V(S)=L(S)$ is generally proved for all surfaces $S$ of the type of the 2-cell, i.e., for mappings from a 2-cell $Q$, by making use first of the standard inequality $\left(^{*}\right) V(S) \leqq L(S) \leqq \sum_{i} V\left(T_{i}\right)$, and then of the concepts of $F$-equivalence, Dirichlet integral, generalized conformal representation, and topological structure properties of continuous mappings from a 2-cell. All these important concepts, however, are far from the simple geometrical meaning of the equality $V=L$. A direct proof of the equality $V(S)=L(S)$ for all continuous mappings from any arbitrary open set $Q$ is now given. The proof, which is independent of the concepts mentioned above, is based on the existence of everywhere dense rectifiable contours on each surface of finite Lebesgue area (L. Cesari, Bull. Amer. Math. Soc. vol. 57 (1951) p. 168; R. E. Fullerton, ibid. vol. 58 (1952) pp. 57 and 180) and makes systematic use of the inequality $\left({ }^{*}\right)$. (Received October $\left.15,1952.\right)$

\section{Y. W. Chen: Degenerate solutions of quasi-linear partial differen- tial equations in $n$ independent variables.}

Consider equation $A_{i j}\left(p_{1}, \cdots, p_{n}\right) \partial^{2} u / \partial x_{i} \partial x_{j}=0$ with $A_{i j}=A_{j i}$ and $p_{i}=\partial u / \partial x_{i}$. A degenerate solution of the equation is called an $s$-tuple wave if it satisfies $n-s$ relations $p_{\alpha}=F^{(\alpha)}\left(p_{1}, \cdots, p_{s}\right), \alpha=s+1, \cdots, n$. An $s$-tuple wave will be represented by $u=\sum_{s+1}^{n} x_{\alpha} F^{(\alpha)}+\phi+\sum_{1}^{c} p_{k} x_{k}$ where the $n-s+1$ unknown functions $F^{(\alpha)}$ and $\phi$ of $s$ variables $p_{1}, \cdots, p_{s}$ satisfy $(n-1) ! /(n-s) !(s-1) !$ partial differential equations in $s$ independent variables. The problem of finding simple waves $(s=1)$ is under-determined (not enough equations) unless $n=2$, and that of finding $s$-tuple waves with $s>2$ is over-determined (too many equations). Only when $s=2$ are there as many equations as unknown functions. In this case of double waves the system of $n-1$ equations has the form $C_{11} F_{11}^{(\alpha)}+2 C_{12} F_{12}^{(\alpha)}+C_{22} F_{22}^{(\alpha)}=0$ where $F_{i j}^{(\alpha)}=\partial^{2} F^{(\alpha)} / \partial p_{i} \partial p_{i}$, and the $C_{i j}$ depend on $p_{1}, p_{2}, F^{(3)} \ldots F^{(n)}$ and their first partial derivatives with respect to $p_{1}$ and $p_{2}$. When $F^{(\alpha)}$ are obtained, one finds $\phi$ by solving $C_{11} \phi_{11}+2 C_{12} \phi_{12}+C_{22} \phi_{22}=0$, $\phi_{i j}=\partial^{2} \phi / \partial p_{i} \partial p_{j}$. Each coefficient $C_{i j}$ is the same in all $n-1$ equations. Such system, if hyperbolic, admits solutions for initial value problems, as is well known. The method used here to derive the differential equations for $F^{\alpha}$ is an application of contact transformations together with suitable separation of variables. Special examples of degenerate solutions are Meyer-Prandtl flows in the plane, conical flows in the space, and others. (Received October 14, 1952.)

88. S. D. Conte and W. C. Sangren: An eigenfunction expansion associated with a pair of first order differential equations. 
Consider the equations $u^{\prime}(x)-[\lambda+a(x)] v(x)=0$ and $v^{\prime}(x)+[\lambda+b(x)] u(x)=0$ where $a(x), b(x)$, and their derivatives are continuous and $L(0, \infty)$. Further consider those solutions $(u, v)$ which satisfy the boundary condition $u(0) \cos \alpha+v(0) \sin \alpha=0$ and the condition $\int_{0}^{\infty}\left(|u|^{2}+|v|^{2}\right) d x<\infty$. Then the values of $\lambda$ for which such solutions exist form a continuous spectrum extending over the entire real axis. Two arbitrary functions $[f(x), g(x)]$ which satisfy the boundary condition $f(0) \cos \alpha+v(0) \sin \alpha=0$ may under appropriate restrictions be represented by the generalized Fourier integrals $f(x)=\int_{-\infty}^{\infty} h(\lambda) u(x, \lambda) d \lambda$ and $g(x)=\int_{-\infty}^{\infty} h(\lambda) v(x, \lambda) d \lambda$. The methods used are patterned after those of E. C. Titchmarsh (Eigenfunction expansions associated with second order differential equations, Oxford, 1946). (Received September 18, 1952.)

\section{R. E. Fullerton: The structure of $L$ cones and a geometric char- acterization of L-spaces.}

Let $X$ be a linear topological space and let $C$ be a cone with vertex at the origin which is the closed convex hull of its set of extreme rays. $C$ is a $C$-cone if for every $x \in X$ there exists a $y \in X$ with $C \cap(x+C)=y+C . C$ is an $L$-cone if there exists some $x \in X, x \notin C \cup(-C)$, and a $y \in X$ such that $C \cap(x+C)=y+C$. It is shown that an $L$-cone is either a $C$-cone or the union of two $C$-cones with a common face and that a necessary and sufficient condition that a Banach space $X$ whose unit sphere $S$ is the closed convex hull of its extreme points be a space of integrable functions is that for any extreme point $v \in S$ there exists an $L$ cone $C$ with $S=(v+C) \cap(-v-C)$. (Received October 15, 1952.)

\section{Michael Golomb: A function-theoretic method for computing} eigenvalues and eigenfunctions. Preliminary report.

Suppose $f(z)$ is regular analytic for $|z| \leqq r$ except for the simple poles $\lambda_{1}, \cdots, \lambda_{n}$, $0<\left|\lambda_{i}\right|<r$. It is well known how the product $\Pi \lambda_{i}$ can be obtained as the limit of a quotient of determinants involving the coefficients $a_{\nu}=f^{(\nu)}(0) / \nu$ !. Similarly a limit involving the symmetric functions of $\lambda_{1}, \cdots, \lambda_{n}$ beside the coefficients $a_{\nu}$ is found for the sum of the residues of $f(z)$. These results are used to compute the eigenvalues and eigenprojectors of a bounded normal linear operator $A$ in a Hilbert space. Let $\mathcal{A}$ be the Banach algebra over the complex field generated by $A$ and $\tau(X)$ a complex-valued bounded linear functional on $\mathcal{C}$. Put $R(\lambda)=A(1-\lambda A)^{-1}$ and assume the part of the spectrum of $A$ for which $|z| \geqq r^{-1}$ consists of the eigenvalues $\lambda_{1}^{-1}, \cdots, \lambda_{n}^{-1}$, the corresponding eigenprojectors being $P_{1}, \cdots, P_{n}$. Then $f(z)=\tau(R(z))$ has simple poles at $\lambda_{1}, \cdots, \lambda_{n}$ if $\tau\left(P_{i}\right)=0$, and $f^{(\nu)}(0) / \nu !=\tau\left(A^{\nu+1}\right)$. Hence, $\Pi \lambda_{i}$ can be expressed as a limit involving the numbers $\tau\left(A^{\nu}\right)$ and $\sum P_{i}$ can be expressed as a limit involving the symmetric functions of $\lambda_{1}, \cdots, \lambda_{n}$ beside the operators $A^{\nu}$. Specialization of the function $\tau$ leads to various iteration formulae for the eigenvalues, some known, some new. Application is made to eigenvalues and eigenfunctions of integral kernels. (Received October 14, 1952.)

\section{1t. Michael Golomb: A general Fredholm theory for linear equa- tions. Preliminary report.}

Suppose $A$ is a bounded linear operator in a complex Banach space, $A$ is the Banach algebra generated by $A$, and $\tau(X)$ is a complex-valued bounded linear functional on $\mathcal{A}$. Define the numbers $n ! d_{n}=\sum(-1)^{r} \tau\left(A^{p_{1}}\right) \cdots \tau\left(A^{p_{r}}\right)$ where the sum extends over all permutations of $1,2, \cdots, n$, and $p_{1}, \cdots, p_{r}$ are the orders of the cycles constituting the permutation. Likewise define the operators $n ! D_{n}=\sum(-1)^{r}$ 
$\cdot \tau\left(A^{p_{1}}\right) \cdots \tau\left(A^{p_{r}}\right) A^{p}$ with the sum extending over all permutations of $0,1, \cdots, n$; $p$ being the order of the cycle containing 0 . The functions $d(\lambda)=\sum d_{n} \lambda^{n}$ and $D(\lambda)$ $=\sum \lambda^{n} D_{n}$ satisfy the identities $D(\lambda)(1-\lambda A)=(1-\lambda A) D(\lambda)=d(\lambda) A$, hence function like the fundamental determinant and minors of the Fredholm theory of integral equations. Conditions on the functional $\tau$ are investigated under which $d(\lambda), D(\lambda)$ are entire functions and under which the poles and residues of $D(\lambda) / d(\lambda)$ are the eigenvalues and eigenprojectors of $A$. (Received October 14, 1952.)

\section{J. H. B. Kemperman: Exact solutions for a certain class of in- tegral equations.}

Let $a=a(x)$ and $F(x, y)$ be defined for $x, y \in E$, with $F(x, y)=\sum_{1}^{p} d_{k}(x) F_{k}(y)$ if $a(x) \neq 0$ and $a(y) \neq 1$ (restricted degeneration). Let $U g=\int_{E} g(y) d F(x, y)$ and $T g$ $=a U g$. Let $(1-\lambda U) g_{i}=h_{i}(i=1, \cdots, q ; q \geqq p)$, where $\lambda$ is a scalar and where $h_{i}$ is linearly dependent on the set of $p$ functions $d_{k}=d_{k}(x) \quad(k=1, \cdots, p)$. Finally, let $(1-\lambda U) g_{0}=h_{0}$. Then, given a certain independence condition, the constants $\alpha_{1}, \cdots, \alpha_{q}$ can be found such that the function $g=a\left(g_{0}+\alpha_{1} g_{1}+\cdots+\alpha_{q} g_{q}\right)$ satisfies $(1-\lambda T) g=a h_{0}$. This principle sometimes enables us to construct a solution of the latter equation. An important application is that where $E$ is the real axis, $a(x)=1$ for $0<x<e, a(x)=0$ otherwise (eventually, $c=+\infty)$. Assume that $F(x, y)=F(y-x)$, where, for $x<0, F(x)$ is a finite sum of terms $c_{j} x^{m_{j}} e^{\lambda_{j x}}\left(c_{j}, \lambda_{j}=\right.$ complex numbers, $m_{j}=$ integer $\geqq 0$ ). Only if $c<\infty$ we require a similar condition for $x>0$. Further, suppose that $\phi(t)=\int_{-\infty}^{\infty} e^{t x} d F(x)$ converges for $\operatorname{Re}(t)=t_{0}$ and, moreover, that $\phi(t)$ can be extended as an analytic and single-valued function in a certain open and connected region $D$ (if $c<\infty, \phi(t)$ is a rational function). If $g_{i}=e^{t x}, t \in D, \phi(t)=1 / \lambda$, and $T g_{i}$ exists, it turns out that $g_{i}$ serves the above purpose even if $U g_{i}$ does not exist (if $\lambda U g_{i}$ exists it is equal to $g_{i}$, thus, $\left.h_{i}=0\right)$. Example: if $F(x)=e^{x}$ for $x<0$ and $\phi\left(t_{1}\right)$ $=\phi\left(t_{2}\right)=1 / \lambda$, the function $g(x)=\left(1+t_{2}\right) e^{t_{2} x}-\left(1+t_{1}\right) e^{t_{1}{ }^{x}}$ satisfies $g(x)=\int_{0}^{\infty} g(y) d F(y-x)$, provided that the latter integral exists. (Received November 12,1952.)

\section{3t. A. J. Lohwater and George Piranian: On the derivative of $a$ univalent function.}

It is known that, if $f(z)$ is analytic and univalent in $|z|<1$, then $\left(^{*}\right) \lim _{r \rightarrow 1}(1-r)^{1 / 2}$ $\left|f^{\prime}\left(r e^{i \theta}\right)\right|=0$ for all $e^{i \theta}$ on $|z|=1$ except for a possible set of linear measure zero (Seidel and Walsh, Trans. Amer. Math. Soc. vol. 52 (1942) p. 141). In view of Beurling's success in cutting down the size of certain exceptional sets associated with a univalent function and its derivative, it is natural to ask whether the exceptional set associated with $\left(^{*}\right)$ can be described in terms of logarithmic capacity. It is proved that there exists in $|z|<1$ a univalent function $f(z)$ such that $\lim _{r \rightarrow 1}(1-r)^{\beta}\left|f^{\prime}\left(r e^{i \theta}\right)\right|$, $0 \leqq \beta<1$, is infinite on a set of positive capacity. This result depends on the following lemma: If $\mu(t)$ is of bounded variation in $(-\pi, \pi)$, and if $t_{0}\left(-\pi<t_{0}<\pi\right)$ is such that $\left.\lim _{h \rightarrow 0}\left(\mu\left(t_{0}+h\right)-\mu\left(t_{0}-h\right)\right) / h^{\alpha}=\infty(0<\alpha \leqq 1) ; h>0\right)$, then the harmonic function $u(r, \theta)=(1 / 2 \pi) \int_{-\pi}^{\pi} K(r, \theta-t) d \mu(t)$, where $K(r, \theta)$ is the Poisson kernel $\left(1-r^{2}\right) /\left(1+r^{2}-2 r \cos \theta\right)$, has the property that $\lim _{r \rightarrow 1}(1-r)^{1-\alpha} u\left(r, t_{0}\right)=\infty$. (Received September 17, 1952.)

\section{Arthur Rosenthal: On functions with infinitely many derivatives.}

For real functions $f(x)$, analytic at $x=x_{0}$, the derivatives at $x=x_{0}$ cannot be chosen quite arbitrarily. It is shown that in the case of infinitely of ten differentiable functions $f(x)$ the values of the successive derivatives at $x=x_{0}$ can be assigned in an entirely 
arbitrary manner. The proof uses polynomials which are closely related to the Hermite polynomials. (Received October 13, 1952.)

95. C. J. Titus and G. S. Young: The maximum modulus principle for certain differential systems.

Generalizing the work of G. Welsky, A. Krowade, and others, the authors prove the following. Let $u_{i}=u_{i}\left(x_{1}, \cdots, x_{n}\right), i=1, \cdots, n$, be a mapping of class $C^{1}$ defined on a domain $D$ in $E^{n}$. Suppose that the Jacobian of $\left\{u_{i}\right\}$ does not change sign in $D$ and that the function is constant on every connected open set on which the Jacobean vanishes. Then each $\left|u_{i}\right|$ takes its maximum on the boundary of every open set with a compact closure in $D$. Methods are those of the authors' paper (Michigan Mathematical Journal vol. 1 (1952) 89-93). (Received October 15, 1952.)

\section{Applied Mathematics}

\section{C. L. Dolph: On the Timoshenko theory of the transverse vibration of beams.}

The Timoshenko beam equations (London Philosophical Magazine (1921)), which include correction terms for shear and rotary inertia, are investigated by means of solutions sinusoidal in time. A two term relation, valid independent of the uniformity of the beam, is derived and shown to imply a type of orthogonality of the spatial modes under all the simplest boundary conditions. For the special case of the uniform hinged-hinged beam the normal modes are determined explicitly and a general boundary and initial value problem solved by a superposition of them. The coefficients in these series are determined by the two term orthogonality relation and shown to be equal to Fourier coefficients. Such a solution exists although the given system is not separable without the additional assumption of a sinusoidal time behavior and although this assumption does not appear a priori to yield sufficiently many such modes to satisfy the four initial conditions. However, each spatial part is in fact paired with two functions sinusoidal in time, with different frequencies so that the resulting eigenvectors are orthogonal in the above sense. Universal curves for this case are compared with those computed electronically for the uniform free-free beam where it is not known that the sinusoidal assumption is sufficient. (Received March 24, 1952.)

\section{Y. L. Luke: Mechanical quadrature near a singularity.}

Purpose of paper is to present coefficients to facilitate computation of $\int_{0}^{n h} x^{-1 / 2} f(x) d x$. Coefficients are derived on basis of writing the Lagrangian polynomial which pre cisely fits $f(x)$ at the points $f(r h), r=0,1,2, \cdots, n$. Exact coefficients are given for $n=1(1) 10$. Integration formula is exact if $f(x)$ is a polynomial of degree $n$. The remainder term for the $(n+1)$ point formula is derived after the manner of W. E. Milne (The remainder in linear methods of approximation, Journal of Research, National Bureau of Standards, vol. 43 (1949) pp. 501-511). He writes $R_{n}=\int_{0}^{n h} f^{(n+1)}(s) G(s) d s$. Mean value theorem is applicable if $G(s)$ is of constant sign in the interval of integration. This is so for the even point formulae. For the odd point formulae, $G(s)$ vanishes at one point in the open interval of integration. Let $\xi$ be the point. For each $n$ exact values of $\int_{0}^{n h} G(s) d s$ are tabulated. For the odd point formulae, values of $\xi$ and $\int_{0}^{\xi} G(s) d s$ are also presented, mostly to $6 d$. (Received October 10,1952.)

98t. K. O. May: Note on the complete independence of the conditions for simple majority decision. 
A method of group decision may be characterized by specifying a function giving group choice in terms of the choices of the individuals making up the group. In a previous paper ( $A$ set of independent necessary and sufficient conditions for simple majority decision, Econometrica, October 1952) the author showed that the familiar method of deciding between two alternatives by simple majority vote is characterized categorically by four conditions on the group decision function. These conditions are here shown to be completely independent by exhibiting functions satisfying every possible combination of their truth values. (Received October 14, 1952.)

99t. L. E. Payne: Axially symmetric problems in elasticity. II. The crack problem.

This paper gives a new approach to the problem of determining the distribution of stress in an infinite elastic medium when a normal pressure is applied to the faces of a disk-shaped crack (see, I. N. Sneddon, Fourier Transforms, 1951). Let the plane of the crack be the plane $x=0$ and let $y$ be the radial coordinate. The problem then amounts to the determination of the stress distribution throughout the medium with the conditions that on the plane $x=0$ all shearing stresses vanish, for $y>b$ no displacement normal to this plane exists, and for $y<b$ either the normal stress or nor$\mathrm{mal}$ displacement be prescribed. In this paper the problem is reduced to that of determining the potential of a magnetic disk. By use of curvilinear coordinates the customary tedious procedure of solving a pair of dual integral equations can be eliminated. This new method of formulation, which is the same as that employed in treating the punch problem (L. E. Payne, Axially symmetric problems in elasticity. I. The punch problem, Bull. Amer. Math. Soc. Abstract 58-6-681) shows that the crack and punch problems are actually equivalent to certain half space torsion problems. (Received October 3, 1952.)

\section{GEOMETRY}

100. P. O. Bell: On certain limit points determined by means of a prescribed metric at a generic point of a curve.

Let $x(-\sigma), x(0), x(\sigma)$ denote neighboring points of a space curve $C$ in ordinary projective space. Let $\pi_{\mu}$ denote an arbitrary plane passing through the tangent to $C$ at $x(0)$. Let $P(\sigma, \mu)$ denote the point of intersection of the line joining $x(-\sigma), x(\sigma)$ with the plane $\pi_{\mu}$. As $\sigma$ tends to zero the point $P(\sigma, \mu)$ approaches a limit point $P(0, \mu)$ which is a point of the tangent to $C$ at $x(0)$, and it is distinct from $x(0)$ if, and only if, $\pi_{\mu}$ osculates $C$ at $x(0)$. If $\pi_{\mu}$ osculates $C$ at $x(0)$, the limit of the line joining $x(0)$ and $P(\sigma, \mu)$ is a line distinct from the tangent to $C$ at $x$. Use is made of these concepts to characterize geometrically: (1) A covariant reference frame for $C$ associated with an element of projective arc length, (2) the projective normal of an analytic surface, and (3) the curvature and torsion of a curve $C$. The last of these characterizations is as follows: Let $\phi$ denote the angle in the positive sense from the osculating plane to the plane $\pi_{\mu}$, and let $\Delta$ denote the directed distance from $x(0)$ to $P(0, \mu)$. Tan $\phi$ is proportional to $\Delta$ if, and only if, $k^{1 / 3} d s / d \sigma=$ const., $s$ denotes arc length, $k=$ curvature. In this case $\tan \phi=T \Delta / 3, T=$ torsion. (Received October 14, 1952.)

\section{Oswald Wyler: Incidence geometries.}

The lattice $L$ of flats of a geometry satisfying Hilbert's axioms of incidence is an exchange lattice (MacLane, Duke Math. J. vol. 4 (1938) pp. 455-468), and the quotient lattice $L / P$ is modular for every point $P$ of $L$. These two properties furnish 
a lattice-theoretic characterization of incidence geometries of any dimension. Two systems of axioms for such geometries of dimension $\geqq 3$ are given. The first one, with points and planes as primitive notions, is valid for any dimension; the second one, with points and hyperplanes as primitive notions, is valid for finite-dimensional geometries. An incidence geometry is called irreducible if every pencil of lines contains at least three lines. It is shown that any irreducible incidence geometry of dimension $\geqq 4$ can be embedded into a projective geometry of the same dimension by the construction of bundles. (Received October 7, 1952.)

\section{LOGIC AND Foundations}

\section{2t. J. C. E. Dekker: Productive sets.}

A collection of non-negative integers is called a set. Let $\Phi(n, x)$ be the partial recursive function discussed by Kleene [Trans. Amer. Math. Soc. vol. 53 (1943), p. 58] which generates all partial recursive functions of one variable. Following Rice [Classes of recursively enumerable sets and their decision problems, to be published] we use $\Phi(n, x)$ to characterize recursively enumerable (r.e.) sets. Let $\omega_{n}$ denote the range of $\Phi(n, x)$, then $\left\{\omega_{n}\right\}$ is a sequence of r.e. sets in which every r.e. set occurs at least once. The set $\alpha$ is called productive if there exists a partial recursive function $p(n)$ such that $\omega_{n} \subset \alpha$ implies: (1) $p(n)$ is defined, (2) $p(n) \in \alpha-\omega_{n}$. Every such function $p(n)$ is called a productive function of $\alpha$. Let Dom $\alpha$ denote the set of all $n$ such that $\omega_{n} \subset \alpha$. The set $\pi$ is called a productive center of the productive set $\alpha$ if $\pi=p(\operatorname{Dom} \alpha)$ for some productive function $p(n)$ of $\alpha$. Theorems. A productive set is not r.e., but has an infinite r.e. subset. There exists a productive set among whose productive centers denumerably many are mutually disjoint. If $\pi$ is a productive center of the productive set $\alpha$ and $\pi \subset \beta C \alpha$, then $\beta$ is a productive set with a productive center included in $\pi$. (Received October 9, 1952.)

\section{3t. J. C. E. Dekker: Two notes on recursively enumerable sets.}

A collection of sets of non-negative integers is called a class. $Q$ denotes the class of all sets which are finite or empty, $P$ the class of all sets which have a finite or empty complement, $E$ the class of all recursive sets, $F$ the class of all recursively enumerable (r.e.) sets. Post introduced the following concepts: simple set, hypersimple set, oneone reducible, many-one reducible [Bull. Amer. Math. Soc. vol. 50 (1944) pp. 284316]. The complete set defined by Post [loc. cit. p. 295] is denoted by $\kappa$, the simple set defined by Post [loc. cit. p. 298] by $\zeta$. $H$ denotes the class of all r.e. sets whose complement is productive [see the preceding abstract], $Z$ the class of all simple sets, $Z_{0}$ the class of all hypersimple sets, $J$ the class $(F-E)-(H+Z)$. Theorem of the first note. Both $Z+P$ and $Z_{0}+P$ are dual ideals in $F$. Theorem of the second note. There exists a set $\nu$ belonging to $J$ whose degree of unsolvability relative to one-one reducibility lies between that of $\zeta$ and that of $\kappa$, while its degree of unsolvability relative to many-one reducibility is the same as that of $\zeta$. (Received October 9, 1952.)

\section{4t. J. C. E. Dekker: Productive classes.}

For notations see the two preceding abstracts. Let $\Omega_{n}$ denote the class of all r.e. sets over which $\omega_{k}$ ranges if $k$ ranges over $\omega_{n}$. The subclass $A$ of $F$ is called r.e. if $A=\Omega_{n}$ for some $n$. The author proved in an earlier (not yet published) paper that the classes $P, Q, E, F$ are r.e. The subclass $A$ of $F$ is called productive if there exists a partial recursive function $p(n)$ such that $\Omega_{n} \subset A$ implies: (1) $p(n)$ is defined, (2) $\omega_{p(n)} \in A-\Omega_{n}$. Every such function $p(n)$ is called a productive function of $A$. Let 
Dom $A$ denote the set of all $n$ such that $\Omega_{n} \subset A$. The class $T$ is called a productive center of the productive class $A$ if there exists a productive function $p(n)$ of $A$ such that $\omega_{p(n)}$ ranges over $T$, when $n$ ranges over $\operatorname{Dom} A$. Theorem. If $T$ is a productive center of the productive class $A$ and $T \subset B C A$, then $B$ is a productive class with a productive center included in $T$. Let $D$ denote the class $E-(P+Q)$. A subclass $A$ of $F$ is called creative if $A$ is r.e. and $F-A$ productive. Theorem. The class $F-Q$ is productive; among its productive centers at least one is included in $D$, at least one in $H$ and at least one in $J$. Corollaries. $D, H, J$ are productive. $Q$ and $E$ are creative. (Received October 9, 1952.)

\section{P. C. Hammer: Generalized extreme points.}

Let $M$ be a set, $S_{1}$ and $S_{2}$ classes of subsets of $M_{1}$, and $f$ a function with domain $S_{1}$, range $S_{2}$, where $S_{1}$ does not contain the null set, $N$. A subset $X$ of $M$ is said to be $f$-closed if $X \supset Y \in S_{1}$ implies $X \supset f(Y) . f$ is canonical if $Y \cap f(Y)=N$ for every $Y \in S_{1}$. Every $f$ has an equivalent canonical function. Consider only canonical functions. $x$ is an extreme point of an $f$-closed set $X$ if $X \supset Y \in S_{1}$ implies $x \in \in^{\prime} f(Y)$. A set $Z$ is $f$-discrete if $Z \supset Y \in S_{1}$ implies $Z \cap f(Y)=N$. The set of extreme points of an $f$-closed set is $f$-discrete. Every subset of an $f$-discrete set is $f$-discrete. Every $f$-discrete set contains the extreme points of its $f$-closure. An $f$-closed set minus its extreme points is $f$-closed. (Received September 29, 1952.)

\section{TOPOLOGY}

\section{J. G. Hocking: Homeomorphic approximations to monotone} maps on noncompact 2-manifolds. Preliminary report.

Using a suitable definition of a 1-monotone map of a (noncompact) locally compact (separable metric) space, the following theorem is established: Let $f$ be a uniformly continuous map of a (not necessarily compact) ulc ${ }^{1}, 2$-manifold $M^{2}$ (with or without ulc boundary) onto itself. Then $f$ can be approximated uniformly by homeomorphisms of $M^{2}$ onto itself if and only if the map $f$ is 1 -monotone. The methods of proof are largely those of G. T. Whyburn and G. S. Young. (Received October 10, 1952.)

\section{J. D. McKnight, Jr.: Characterizations of function-rings. Pre-} liminary report.

Let $C(X)$ be the ring of all continuous real-valued functions over a compact Hausdorff space $X$. Among the characterizations of $C(X)$ obtained by using the Stone topology for special classes of ideals, one is found by noting that the following conditions are fulfilled: (1) $C(X)$ is semi-simple; $(2) C(X)$ is an algebra with unity over the real field; (3) there is an isomorphism of $C(X)-I$ into the real field for every primitive ideal $I$; (4) the space of maximal (primitive) ideals of $C(X)$ is a Hausdorff space, i.e., if $I_{1}$ and $I_{2}$ are different maximal ideals, there exist ideals $J_{1}$ and $J_{2}$ such that $J_{i} \subset I_{i}, J_{i}\left\lceil I_{i}\right.$ for $i \neq j$, and $J_{1} \cap J_{2}=0$; (5) the elements of $C(X)$ satisfy a boundedness property: there is a real number $a$ such that for any $b \in C(X)$ there is another element $c \in C(X)$ such that $n(a 1)-b=c^{2}$ for some natural number $n ;(6) C(X)$ has no proper extension satisfying (1) - (5) which preserves the topology on the space of maximal ideals, i.e., if $C(X) \cong C \subset B, B$ satisfying (1) - (5), and if the correspondence $I \rightarrow I \cap C$ is one-one from the set of maximal ideals of $B$ to the set of maximal ideals of $C$ and $J \cap C C I \cap C$ implies $J C I$ for ideal $J$ and maximal ideal $I$ of $B$, then $B=C$. (Received October 14, 1952.) 
108. P. S. Mostert: Fiberable spaces. I. Preliminary report.

Seven classes, $F_{0}, \cdots, F_{6}$, of fiberable spaces are defined, where $F_{6}$ is the class of fibre bundles, and an element of $F_{0}$ is a triple $\{X, B, \pi\}$ where $X$ and $B$ are topological spaces, and $\pi$ is a continuous, open map of $X$ on $B$. A partial cross section w.r.t. a class $K$ of subsets of $B$ is a mapping $f$ of some set $A$ of $K$ into $X$ such that $\pi f(a)=a$. It is the object of this and subsequent papers to study the interrelations of these concepts. In particular, criteria are discussed for the existence of partial cross sections when the class $K$ consists of the arcs of $B$. (Received October 16,1952.)

109. M. E. Shanks: Homology and rings of continuous functions. Preliminary report.

Homology groups of an associative ring $\Re$ have been defined by S. T. Hu in his paper, Homology groups of a ring, Bull. Calcutta Math. Soc. vol. 42 (1950) pp. 123130 , where he shows that if $\Re=C(X)=$ the ring of all real continuous functions over a compactum $X$, then his homology groups are the Alexander-Kolmogoroff groups of $X$. In this paper the above result is shown to be valid for: (1) a larger class of spaces, (2) certain proper subrings of $C(X)$, (3) much smaller subrings if $X$ is a polytope. In addition the whole theory is recast in a form depending on the support of $f \in C(X)$ instead of on the zero set of $f$ as was done by $\mathrm{Hu}$. Finally, a comparison is made between the above theory and the theory of differential forms on manifolds. (Received October 17, 1952.)

J. W. T. Youngs, Associate Secretary 